\title{
Delivering critical science by enabling small interplanetary missions beyond Mars
}

Authors (in order of contribution):

\begin{abstract}
Alyssa Rhoden
Jacob Englander

Elisabeth Morse

Gabe Benavides

Kevin Walsh

Lucy F. Lim
\end{abstract}

Co-signers (in alphabetical order):

Paul Byrne

Julie Castillo-Rogez

Devon Burr

Joshua Emery

Craig Hardgrove

Terry Hurford

Sam Howell

Rob Lillis

Keith Noll
Southwest Research Institute, Boulder

NASA Goddard Space Flight Center

Northrup Grumman Space Systems

NASA Glenn Research Center

Southwest Research Institute, Boulder

NASA Goddard Space Flight Center

\author{
North Carolina State University \\ NASA Jet Propulsion Laboratory \\ Dept. of Astronomy and Planetary Science, NAU \\ Dept. of Astronomy and Planetary Science, NAU \\ School of Earth and Space Exploration, ASU \\ NASA Goddard Space Flight Center \\ NASA Jet Propulsion Laboratory \\ Space Sciences Lab, Berkeley \\ NASA Goddard Space Flight Center
}

Lead author contact information:

Alyssa Rhoden

Southwest Research Institute, Boulder

alyssa@boulder.swri.edu 
Introduction

The main asteroid belt is rich with potential targets for spacecraft investigations. Earth-based observing assets have shown that the composition of bodies in the main belt change with distance from the Sun (e.g. DeMeo and Carey, 2014; Figure 1) - from rocky, silicate-rich Stypes dominating the inner main belt to dark, potentially volatile-bearing P-types and D-types dominating the outer main belt as well as the small body populations at Jupiter and Saturn (e.g. Trojan and irregular satellites). The diversity of asteroids requires many objects to be visited with spacecraft in order to understand the nature of different asteroids, how they are related to small bodies on more distant orbits, the conditions that led to their formation and scattering, their role in altering the surfaces and compositions of planets and moons, and why some asteroids appear to be active.

Unlike the rockier S-types that dominate the inner main belt, more distant asteroids tend not to have spectral features in the visible wavelength range (DeMeo et al., 2009; Reddy et al., 2015). Obtaining more diagnostic near- and mid-infrared observations from Earth is challenging due to the limited solar radiation at long distances and the complicating effects of Earth's atmosphere. Unfortunately, it is precisely these dark, distant objects that have the most to teach us about the early evolution of our solar system, as they are thought to have originated not where we find them now but throughout the outer solar system, including as far away as the Kuiper Belt (Levison et al. 2009, Walsh et al. 2011, Warren et al. 2011, Raymond and Izidoro et al. 2017). The main belt also contains asteroid families of various types (e.g., Nesvorny et al. 2015; Masiero et al. 2015);

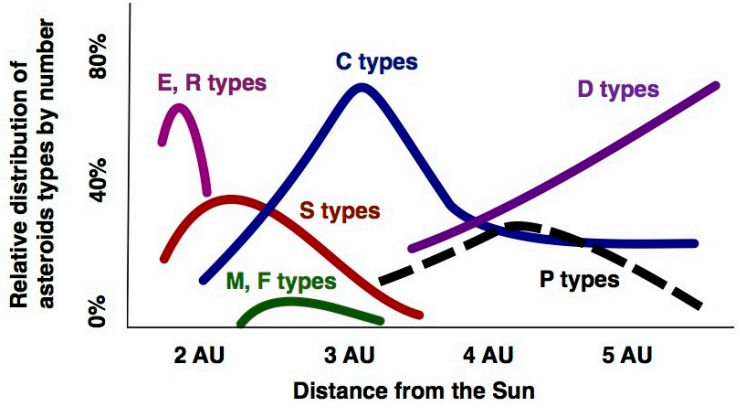

Figure 1: Asteroid compositions change with distance from the Sun. Small satellites have the potential to visit these targets with more frequency and at much lower cost than larger missions. Technology investment and modest changes to the structure of small mission programs can enable exploration of all asteroid types, vasty expanding our knowledge of small bodies and the solar system processes that led to their formation and distribution. Image credit: Gradie and Tedesco (1982), colorized by Newton (2014). in situ exploration of collisional families can shed light on the original structure of large asteroids and the composition of interior layers, which can be challenging to constrain by other means. Some of the enigmatic active asteroids (or "main belt comets") can be found amidst these families, although they too are typically found in the outer reaches of the main belt (Jewitt et al. 2015).

The completeness of Earth's meteorite collection is unknown. In situ observations at an asteroid can obtain critical measurements of subsurface composition that can be compared directly with meteorites. Definitively tying meteorites to asteroid classes, identifying asteroid classes not represented by meteorites, and expanding the range of known compositional classes present in the solid bodies of our solar system have wide-reaching implications, including understanding compositional mixing in the early solar system, how giant impacts can create the final compositions of moons generated in such impacts, the timing and extent to which volatiles were delivered by asteroids from different small body reservoirs, and the strengths of asteroids with different compositions.

NASA's Discovery and New Frontiers missions can work on long timescales to do grand tours (e.g., Lucy) or very deep studies of one or a few bodies (e.g. Dawn, Psyche, OSIRIS-REx) 
with customized instrumentation. These missions have greatly increased our knowledge of the solar system, as will the current and upcoming small bodies missions. They are also rare, highly competitive, and require a large number of resources to support proposal development.

Within the last decade, NASA's investment in small satellite technologies has resulted in many advances that can enable significant science return from ride-share compatible, small satellites. For example, a small-scale neutron detector has been developed for the LunaH-Map mission, a miniaturized NIR point spectrometer has been developed for the Lunar IceCube mission, and the MarCO mission demonstrated high data rate communication from Mars with a novel Reflectarray in a set of $6 \mathrm{u}$ CubeSats. The Janus mission to fly by two binary near-Earth asteroids at $\sim 1 \mathrm{AU}$ and the ESCAPADE mission to Mars are addressing challenges posed by traveling beyond the Earth-Moon system with an ESPA-limited, ride share spacecraft. @fWith these advances, we are incredibly close to accessing the main belt, with its numerous potential targets, and exploring these bodies with instrumentation that can probe from the deep interior to the surface, within the constraints of the current SIMPLEx program. These missions provide many more opportunities for exploration and a cost-effective way of connecting the targets of Discovery and New Frontiers missions with similar bodies closer to home, leveraging all we have learned in those programs.

Unfortunately, the current suite of NASA mission programs for planetary science rules out much of the asteroid science that could be achieved using small spacecraft, particularly those that can ride-share with larger missions. Ride shares add value to every NASA launch, expanding the scope of achievable science. Addressing the technology gaps limiting small spacecraft missions beyond Mars, as well as the programmatic limits currently imposed, could usher in a new era of low cost exploration to the asteroid belt and feed forward into similar cost missions to even more distant targets.

\section{Barriers posed by technology}

The first barrier to small satellite exploration beyond Mars is a lack of hardware technologies for deep space small sat applications, many of which are detailed below. Reaching the main belt within a small rideshare package typically requires a low-thrust trajectory with significant total Delta- $\mathrm{V}$ capability, which in turn is a major spacecraft system driver. Many technology requirements necessarily differ between near-Earth orbital and deep space environments. Thus, there is a critical need for NASA to identify key technologies and provide resources to raise their TRL by developing and flight qualifying small spacecraft technologies for deep space ahead of mission need. Otherwise, the diversity, the cadence, and the cost of cutting-edge deep space science will continue to lag the progress of commercial industry in near-Earth orbits.

Propulsion. Rideshare opportunities and low-cost launch vehicles have redefined access to space, but the preponderance of these launches deliver small spacecraft to near Earth orbits. Increasing the cadence of low-cost small spacecraft missions to deep space requires a technological leap in sub-kilowatt electric propulsion systems to ensure highly capable on-board propulsion options. High-propellant throughput, flexibility in operational power, and long life in a deep space environment are key capabilities needed to enable deep space missions. The state-of-the-art small spacecraft propulsion systems are largely optimized to serve the emerging low Earth orbit (LEO) market; commercial LEO missions largely steer these technology developments toward ultra-low cost solutions with limited capability. Missions that propose using LEO propulsion systems for deep space face numerous and potentially 
insurmountable technical challenges. In such circumstances, achieving high-propellant throughput requires integrating multiple propulsion strings in an already volume and mass limited spacecraft. LEO power processing units (PPU) must be redesigned and requalified with more radiation tolerant and better screened components. These PPU redesigns will come at considerable expense to missions and pose significant risk to schedule and budget. Finally, LEO propulsion solutions often sacrifice rigorous testing and physics-based modeling to achieve ultralow cost in exchange for acceptable rates of spacecraft loss. However, reliability of hardware, rigorous testing, and physics-based modeling are critical to demonstrate long life capability and flexibility in the diverse spaceflight environments beyond LEO.

Bridging this technology gap will require NASA to make focused investments in advanced propulsion technologies and maintain those investments through spaceflight qualification. For example, NASA Glenn Research Center (GRC) has made substantial progress advancing subkilowatt electric propulsion technology with the H71M Hall-effect thruster and PPU. The H71M is presently at TRL 4 with test data indicating a capability to offer greater than $8 \mathrm{~km} / \mathrm{s}$ to a small spacecraft. The PPU and thruster are designed to offer a wide power range (100 W to $1 \mathrm{~kW})$ and baseline parts appropriate for the deep space environment. Finally, GRC is actively collaborating with U.S. industry partners to identify new ambitious commercial missions such that these technologies become commercially available in the future and at acceptable cost to NASA funded missions. However, at a current TRL of 4, the technology is not ready for immediate mission infusion, and the required investments are unlikely to come from low-cost science mission budgets. Furthermore, the time required for development and qualification activities exceeds that of a typical low-cost mission.

Power. In order to enable small-sat trajectories beyond Mars, there is a critical need for more flight qualified, large solar power systems, with a small packing factor suitable for ESPA-class spacecraft. These systems must generate sufficient power at large solar distances, within a small mass, with qualification over the range of voltages, currents, and temperatures associated with deep space missions; together with the power electronics to process the varying solar array output along a deep space trajectory.

Telecom. Communications far from Earth require new systems that can simultaneously meet the need for deep space mission data rates (particularly for short duration missions) and a small form factor. For flyby missions, low data return rates can mean long mission durations. For small-body orbiters, higher rates are required to ensure data from high orbits can be accounted for in planning for lower orbit science. In either case, the option to close the link with highpower travelling wave tube amplifiers (TWTA) adds too much difficulty in cost, power, mass, and volume accommodation for small sats. Telecom hardware options of suitable form factor for small sats, that are also capable of deep space links, do not exist with lifetimes consistent with the duration of low-thrust trajectories to main belt asteroids, which call for 3 to 6 year mission durations.

Parts qualification and lifetime. While COTS avionics can achieve capable missions to LEO, the Moon, or even Mars flyby, they do not have the space environment lifetime commensurate with such mission durations. Higher-cost parts remain necessary to enable main belt science (and beyond). While developments are being made in all of these areas, many subsystems and components have not been tested over the lifetimes needed for lengthy deep space travel. In addition, there are limited applications for deep space enabling technologies within the private sector making it unlikely that commercial investment will generate solutions. 
The Iris radio development by JPL was a good example of NASA development now passed to the commercial sector, making it available at much lower cost. Similar investments and licensing agreements could be made in order to stimulate development and maturation of cost effective technologies. We encourage NASA to invest in the technologies critical for low cost, deep space, small sat missions, either directly or in partnership with commercial vendors, to ensure expediency, applicability, and maturity.

While we have focused here on the unique scientific value of targets beyond Mars, the same challenges affect missions to Mars and Venus, limiting the potential science that can be done at those targets. For example, at Mars, eclipses complicate the power strategy. At Venus, only flyby missions have, so far, been shown to fit within the SIMPLEx cost cap. In other words, addressing the barriers described above can enable all deep space missions, expanding the science return of low cost, small satellite missions and reducing the cost of such missions over time through the development and maturation of common technologies.

\section{Barriers posed by SIMPLEx requirements}

Several constraints/requirements within previous SIMPLEx AOs, pose particular challenges to missions beyond Mars:

Cost structure. The inclusion of Phase E costs within the SIMPLEx cost cap reduces the potential breadth and depth of science investigations with distance from Earth. The personnel required to support a long flight time does not scale proportionally down with the size of the spacecraft, especially for low-thrust missions for which the cruise cannot be a dormant period. Allocating the required funds for Phase E within the very small SIMPLEx cost cap limits the instrumentation and personnel budgets, reducing the competitiveness of long duration missions. Beyond even Phase E and hardware costs, there is a minimum set of labor costs associated with any planetary mission, which does not scale proportionally with cost cap: program management, systems engineering, trajectory design, low-thrust electric propulsion system integration, link analysis, thermal modeling, optical navigation, low-body orbit analysis, instrument viewing geometries, environmental requirements, are all elements of work that are unique to each planetary mission. Such fixed costs must be incurred even if using contributed instruments and product-line small satellites, reducing how much innovation can fit within a SIMPLEx cost cap.

Size limits. The previous mass and volume limits within SIMPLEx were based on the original advertised limits for the ESPA ring capability within a 4-m launch vehicle fairing. There is room to relax constraints in both mass and volume that would enable new science within rideshares. The actual mass capability of each ESPA port is much larger than in previous AOs, and even larger for ESPA Grande, allowing more than a factor of 2 increase over the 180-kg limitation of past AOs. Furthermore, volume is a very hard constraint for rideshares. Future launch vehicle fairings will have 5-m diameters, enabling a much larger volume for rideshares (56 in instead of 38 in) which can enable significantly better science by allowing packaging of solar arrays, science instruments, and high gain antennas. We encourage NASA to revise the size limits within SIMPLEx to account for the current and near-future capacities of launch vehicles.

Rideshare opportunities. Current rideshare opportunities for planetary small sats are limited to dedicated planetary launches, which are rare and expensive. In contrast, geosynchronous transfer orbit (GTO) missions are common, and lunar missions are expected to be frequent in the 2020s. Enabling small sat rideshares to GTO would require an increase in the mass limit within SIMPLEx to accommodate the additional propellant needed for a deep space abled spacecraft to 
escape to an interplanetary trajectory. For lunar rideshares, the mass limit would need to be at least the SIMPLEx limit to accommodate interplanetary missions, and new interfaces, not specific to cube sats, would need to be designed. For missions to the main belt, for example, escape from the Earth-Moon system may be achieved by means of a double lunar gravity assist at the cost of approximately 3 months of flight time. The spacecraft may ride-share with a larger mission either directly to a lunar flyby or alternatively to geosynchronous transfer orbit (GTO) and then grow its orbit via on-board electric propulsion. Enabling rideshare options with flights to the Moon or GTO, would not only increase the science opportunities for each launch, it would also make targets beyond Mars accessible at much higher cadence.

Foreign contributions. The considerable allowance for foreign contributions, which could be very enabling for deep space SIMPLEx missions, is challenging to capitalize on due to the need to encumber costs for back-up technologies from domestic partners. Due to the limited options for many such technologies, cost-effective back-up solutions are not always available, reducing the value of contributions. Requiring encumbrances of the nature expected for a Discovery mission, seems inconsistent with the risk posture of SIMPLEx.

We encourage NASA to alter the next SIMPLEx AO to 1) remove Phase E costs from the cost cap and/or scale the cost cap based on the accessibility of the target, 2) increase the mass and volume limits to those set by the ESPA Grande within a 5-m fairing, and 3) provide a pathway by which foreign contributions can be relied upon (e.g., moving back-up encumbrances outside of the cost cap).

\section{Barriers posed by programmatics}

Programmatic differences between the SIMPLEx and Discovery programs provide an additional challenge to exploration of the main belt and beyond. There is an order of magnitude difference in the cost cap between SIMPLEx and Discovery ( $\$ 55 \mathrm{M}$ versus $\$ 500 \mathrm{M})$, whereas there is only a factor of two difference between the Discovery and New Frontiers cost caps. For reasons outlined above, including hardware selection, mission duration, variety of environments (from power to thermal) and complexity of operations, valuable small sat science missions to targets beyond Mars will necessarily cost more than the current SIMPLEx missions, putting them in the gap between these two programs. Using existing costing tools, Table 1 shows that spacecraft costs for a main belt mission are over the $\$ 55 \mathrm{M}$ cost cap without science instruments, personnel, or any post-launch operations.

The difference in mission class rules out simply submitting a SIMPLEx mission proposal to the Discovery program. In some cases, small satellite components with sufficient testing and heritage to meet Discovery requirements are simply not available. The demands of putting together Discovery proposals for missions also limits the number of such proposals that can be supported by centers, industry partners, and other institutions, and the reduced cadence of Discovery missions (two selections every few years) versus SIMPLEx missions (multiple selections annually) limits the opportunities for low-cost, small-sat missions to be proposed.

At a more fundamental level, the core mission of the SIMPLEx program is to provide a pathway to push the limits of exploration, to design innovative missions that capitalize on new, small-scale technologies that enable lower-cost missions. Hence, these proposed missions are riskier than Discovery missions and rely on technologies that are in earlier stages of development. Discovery is simply not the appropriate program for such missions, yet the cost cap gap provides no other pathway for small-satellite missions to more distant targets. 
Table 1. Small Satellite Cost Model Applied to Electric Propulsion Planetary Small Sat

\begin{tabular}{|lc|}
\hline \multicolumn{2}{l}{ Example SmallSat with H71M Engine } \\
Mass Allocation (kg) & \\
CDH (48 month life) & 2 \\
Power (1500W cap.) & 44 \\
GNC (20 arcsec control) & 4.5 \\
Telecom (10W RF) & 4.5 \\
Propulsion & 28 \\
Structure (Al) & 26 \\
Thermal & 4.5 \\
Harness & 6.5 \\
Total Dry & 120 \\
Xenon Allocation & 60 \\
Total Launch & 180 \\
SSCM Results (C-D): & FY2020 \\
Non-Recurring Total & $\$ 24 M$ \\
Recurring Total & $\$ 33 \mathrm{M}$ \\
\hline TOTAL COST & $\$ 57 M$ \\
\hline
\end{tabular}

With the Explorer program, NASA has been achieving great astrophysics and heliophysics science with multi-year duration missions that satisfy challenging science accommodation, propulsion, and thermal requirements within small, single-string packages. Those are challenges of similar magnitude as planetary missions beyond Mars. By opening a new set of opportunities with a cost cap more aligned with the SMEX or MIDEX programs, NASA would enable missions to a larger suite of planetary destinations than is possible within the current constraints of either SIMPLEx or Discovery. Furthermore, the relative cost difference between SMEX and MIDEX is closer to a factor of two, in contrast to the order of magnitude difference between SIMPLEx and Discovery, resulting in a much larger gap within the planetary mission portfolio than those of astrophysics and heliophysics.

We encourage NASA to consider either 1) establishing a program akin to Astrophysics' SMEX and MIDEX programs that would address the gap in mission cost between SIMPLEX and Discovery, with mission class and proposal requirements appropriate to missions within that cost cap, or 2) including a different set of requirements for missions below $\$ 100 \mathrm{M}$, within the Discovery program, to provide low-cost missions a feasible means of proposal submission for small sat rideshare missions that exceed the SIMPLEx cost cap.

Supporting the next generation of mission PIs

NASA has readily acknowledged the need for diversity within the pool of mission PIs. Exploration is a venture into the unknown, with all new challenges and opportunities; a cohort of scientists, engineers, and technicians with a broad range of experiences, skills, and perspectives can tackle a similarly broad set of challenges, setting us on a course to exceed all limitations. NASA's recent PI Launchpad program provided an excellent opportunity for knowledge sharing and training. The Planetary Science Summer School is also a valuable asset for graduate students and recent graduates. We encourage NASA to continue and expand participation in these programs and to create additional workshops and training programs for people in different career stages. A collection of accessible materials describing the basics of missions (e.g., the different stages of missions, sub-systems, mission classes) would also increase accessibility and promote confidence in potential PIs. 


\section{Conclusion}

There is great value in continuing to select very small missions that would fit well within the current SIMPLEx cost cap. Such missions can increase technical readiness and provide hardware qualification opportunities for small satellite technologies more rapidly, and at lower scientific risk, than if they are placed on larger, more expensive missions as tech demos. Those same technologies can then reduce the costs of small, deep space missions by leveling the playing field in terms of available hardware and leaving more money for science and personnel rather than the NRE required to develop critical technologies. Small satellite missions can also achieve targeted science goals and serve as excellent learning opportunities for scientists who want to develop missions and instrumentation. We, thus, encourage NASA to keep sub-\$50M missions within its mission portfolio while also enabling small satellite missions with broader science objectives and more distance targets that currently fall within the cost cap gap between SIMPLEx and Discovery. Specifically, we suggest 1) cost-neutral modifications to the SIMPLEx AO, 2) continued investment in technology development for deep space small satellite needs, 3) broader training and support for a diverse cohort of mission PIs, and 4) a new pathway to proposal submission for $\sim \$ 100 \mathrm{M}$ missions.

\section{REFERENCES:}

- DeMeo, F. E., Binzel, R. P., Slivan, S. M., \& Bus, S. J. (2009), Icarus, 202, 160.

- DeMeo, F. E., \& Carry, B. (2014), Nature, 505, 629.

- Gradie, J., \& Tedesco, E. (1982), Science, 216, 1405.

- Jewitt, D., Hsieh, H., \& Agarwal, J. (2015), Asteroids IV, 221.

- Levison, H. F., Bottke, W. F., Gounelle, M., Morbidelli, A., Nesvorný, D., \& Tsiganis, K. (2009), Nature, 460, 364.

- Masiero, J. R., DeMeo, F. E., Kasuga, T., Parker, A. H. (2015), Asteroids IV, 323.

- Nesvorný, D., Brož, M., \& Carruba, V. (2015), Asteroids IV, 297.

- Newton, E. (2014) Astrobites. https://astrobites.org/2014/02/01/the-composition-andarchitecture-of-the-asteroid-belt-from-simple-to-complicated-in-just-three-decades/

- Raymond, S. N., \& Izidoro, A. (2017), Icarus, 297, 134.

- Reddy, V., Dunn, T.L., Thomas, C.A., Moskovitz, N.A., Burbine, T.H. (2015), Asteroids IV, 43.

- Warren, P. H. (2011), Earth and Planetary Science Letters, 311, 93.

- Walsh, K. J., Morbidelli, A., Raymond, S. N., O'Brien, D. P., \& Mandell, A. M. (2011), Nature, 475, 206. 